\title{
OFDM PERFORMANCE WITH TWO-RAY MULTIPATH FADING MODEL USING INET FRAMEWORK SIMULATION
}

\author{
Nidal A. Al-Dmour ${ }^{1}$ Hmoud Al-Dmour ${ }^{2}$ Liaqat $\mathrm{Ali}^{3}$ \\ ${ }^{1}$ College of Engineering and Information Technology, Ajman University, UAE, n.aldmour@ajman.ac.ae \\ ${ }^{2}$ Department of Physics, Faculty of Science, Mutah University, JORDAN, hmoud79@mutah.edu.jo \\ ${ }^{3}$ College of Engineering and Information Technology, University of Science and Technology of Fujairah, UAE, \\ 1.ali@ustf.ac.ae
}

\begin{abstract}
IEEE 802.11g uses OFDM (Orthogonal frequencydivision multiplexing) to achieve transmission of data rates up to 54 Mbps. OFDM is a method for transmitting large amounts of digital data over radio frequency and radio wave. This is achieved by splitting the radio signal into smaller sub-signals that are each given different frequencies and transmitted simultaneously to a specific receiver. In wireless communication, the received packet encounters path loss which exists between transmitting antenna and receiving antenna, especially when they are in LOS.Fading is one of the major problems of digital communications, which results from different factors. This paper presents the effect of Two-ray Multipath fading model with the variation of the OFDM data rate transmissions. The experiment was conducted using INET simulation framework. The experiment shows that a high data rate transmission is not always achieving better performance with the existence of Two-ray Multipath Fading model.
\end{abstract}

Key words: Wireless Networks, OFDM, Two-ray Multipath Fading, and INET.

\section{INTRODUCTION}

Wireless technologies have shown a rapid growth during recent years. They include Wireless Wide Area Network (WWAN), Wireless Metropolitan Area Network (WMAN), Wireless Local Area (LAN), Wireless Personal Area Network (WPAN), and Wireless Sensor Network (WSN). This classification of wireless technologies is based on the coverage area and the data rate [1]. WWAN corresponds to digital mobile phone networks, such as Global System for Mobile telecommunications (GSM). Wireless Metropolitan Area Networks (WMANs) are emerging for data transmission in municipal areas. They are limited to fixed point-to-point or point to multipoint connections with restricted mobility [1]. WLAN has been designed to replace and expand legacy LANs. WLAN supports a large number of services, for instance to cover broadband wireless Internet access in hot spot areas, as well as short range serial cable replacement [1]. WLAN enables a fast network installation and easy topology changes. Therefore, WLAN can be established on purely temporary basis, for example conferences, meetings, colleges and universities, and even emergency operations. WLAN also makes possible data networking in places without an existing wired LAN infrastructure, such as in military operations [2].

The IEEE 802.11 is the first standard for WLAN that uses the RF radiation with 1 and 2 Mbps data rates and it is currently the most widely used WLAN [4]. In 1999, the IEEE approved the extension of the previous standard. The new IEEE $802.11 \mathrm{~b}$ extension defines a standard for products of wireless networks working at $11 \mathrm{Mbps}$. The need for wireless access to local networks grows with the number of mobile devices such as notebooks and PDAs, as well as the desire of users to be connected to a network with higher data rate. As a result, other extensions to the standard have been approved such as IEEE $802.11 \mathrm{~g}$, IEEE 802.11a, IEEE 802.11e, and IEEE 802.11n [3].

Wireless Personal Area Network (WPAN) is a wireless network centered around user workspace that typically extends up to $10 \mathrm{~m}$ in all directions [4]. The focus of WPANs is low-cost, low power, short range and very small size. IEEE 802.15.4 is designed for low rate WPANs and it is intended to serve a set of industrial, residential and medical applications with very low power consumption [4].

Wireless Sensor Network (WSN) [5] is composed of large number of sensors and their job is to gather data and forward it to the Internet using various routing protocols. Sensor nodes in WSN are responsible for 
collecting the data and a management node is responsible for data collections. There are two scenarios for data collection: the first scenario, the sensor nodes forward the collected data along other sensor nodes by hopping in regular intervals. The second scenario, management nodes send queries to the sensor nodes and request its data "readings". These queries forwarded across the nodes in WSN using various search mechanisms.

OFDM (Orthogonal frequency-division multiplexing) is a method for transmitting large amounts of digital data over radio frequency and radio wave. This is achieved by splitting the radio signal into smaller subsignals that are each given different frequencies and transmitted simultaneously to a specific receiver. The application of this technique comes with many advantages, one being that it reduces the amount of crosstalk and collisions in signal transmission. In OFDM, the sub-signal is chosen so that the signals are mathematically perpendicular over one OFDM symbol period [7],[8], [9].

Although, OFDM is derived from FDM (frequencydivision multiplexing), OFDM provides much more advantageous properties than traditional FDM technique. OFDM is the key technique that has made $4 \mathrm{G}$ wireless communication possible in the recent years [10]. It is especially important to keep the perpendicular/orthogonal nature of the carriers. This can be done through careful control of the signal. If the orthogonality is not achieved, there can be no generation of the ODFM [11]. Additionally, visible light communication can deliver data through OFDM using an organic solar cells as an energy harvesting receiver or high speed data detector [12].

Orthogonal Frequency Division Multiplexing (OFDM) has been in Wireless communication, Digital Audio Broadcasting (DAB), and High Definition TV (HDTV). OFDM can utilize the system bandwidth for 64 sub carriers where as the spectral efficiency can reach $1.98 \mathrm{Bd} / \mathrm{Hz}[13]$. OFDM is capable to transfer a frequency selective fading channel into a number of flat fading sub-channels.

In 2003, OFDM technique was extended with the release of the $802.11 \mathrm{~g}$. $802.11 \mathrm{~g}$ is one of the modulation standard for wireless LANs and it does work on a frequency of $2.4 \mathrm{GHz}$ with a maximum data rate of $54 \mathrm{Mbps}$. The latest wireless standard 802.11n is using OFDM and a new feature was added which is Multiple Input Multiple Output (MIMO). Table 1 below shows the coding rate and data rate for different modulation techniques.
Table 1: The coding rate and data rate for different modulation techniques.

\begin{tabular}{|l|l|l|}
\hline Modulation & Coding Rate & Data Rates \\
\hline BPSK & $1 / 2$ & $6 \mathrm{Mbps}$ \\
\hline BPSK & $3 / 4$ & $9 \mathrm{Mbps}$ \\
\hline QPSK & $1 / 2$ & $12 \mathrm{Mbps}$ \\
\hline QPSK & $3 / 4$ & $18 \mathrm{Mbps}$ \\
\hline QAM16 & $1 / 2$ & $24 \mathrm{Mbps}$ \\
\hline QAM16 & $3 / 4$ & $36 \mathrm{Mbps}$ \\
\hline OFQAM64 & $2 / 3$ & $48 \mathrm{Mbps}$ \\
\hline QAM64 & $3 / 4$ & $54 \mathrm{Mbps}$ \\
\hline
\end{tabular}

In

wireless communication, the received packet encounter path loss which exists between transmitting antenna and receiving antenna, especially when they are in LOS. The analysis of propagation channel has been investigated in [14], [15]. The received power $\left(P_{r}\right)$ is obtained by using equation 1 . Here is the transmitted power is $\left(P_{T}\right) \cdot G_{T}$ and $G_{R}$ are the transmitting and receiving antenna gain, respectively; $L_{T}$ and $L_{R}$ are the transmitting and receiving path losses. Finally, $L_{P}$ is the propagation channel loss.

$P_{r}=\frac{P_{T} G_{T} G_{R}}{L_{T} L_{R} L_{P}}$

The propagation path loss is composed of three parts as in equation 2 [15]. Where $L_{o}$ stands for the average path loss, $L_{s}$ stands for long term fading, and finally $L_{L}$ stands for short term fading.

$L_{p}=L_{o} L_{s} L_{L}(2)$

Fading is one of the major problems of digital communications, which results from different factors such as refraction multipath, atmospheric reflection, ground reflection, etc [15]. There are three different fading models:

a. Cellular system fading model, such as Nakagami model [16].

b. Point to Point terrestrial LOS link fading model such as Two-ray Multipath Fading Model [17].

c. Satellite Link Fading Model such as ITU-R model[18].

The focus of this paper is on Two-ray Ground Multipath fading model in which the fading results from ground multipath with considering the atmospheric effect. If there are two Antennas, one for transmitting, and the other for receiving. The total field at the receiving antenna is:

$E_{\text {total }}=E_{D}+E_{R} e^{j \Lambda Q}$ 
Where $E_{D}$ is the amplitude of direct (LOS) rays, $E_{R}$ is the amplitude of reflected rays, and the amplitude of phase difference between them.

INET [25] framework has provided simulation models for multipath ground reflection point to point Line of Sight (LOS). This papers analyze the effect of multipath ground reflection on data transmitted between two hosts with various OFDM data transmission rates.

The rest of the papers is organized as follows: Section 2 presents our research methodology. Section3 presents the simulation results and discussions. Finally, the conclusion was presented in section 4 .

\section{RESEARCH METHODOLOGY}

In order to run our simulations to study the two-ray multipath fading model simulation tools were required. There were several network simulation tools. Examples of these tools are: Global Mobile Information Systems Information Library (GloMoSim) [20], Optimized Network Engineering Tools (OPNET) [21], Network Simulator (NS-3) [22], QualNet [26], and Omnet++ [23]. These simulation environment share common characteristics [24]:

a. Deterministic: Each running scenario will produce the same results even though it is repeated several times.

b. Discrete event simulation: Simulation is configured based on sequence of events.

c. Integrated Development Environment (IDE):Friendly user interface to create finite state machines.

d. Model library: having Inter-Network Processors such as switches, routers, and hubs.

GloMoSim (global mobile information system simulator) is used for satellite network simulation environment, wired communication network, and Adhoc networks. GloMoSim can be used to simulate multicast and multihop in wireless communication networks. Additionally, Mobile Ad hoc Network (MANET) is also supported using discrete event simulator.

OPNET modeler (currently known as Riverbed Modeler) is a commercial simulation software provided by Riverbed Technology. OPNET modeler has many libraries to simulate networks with different scenarios. OPNET has a very flexible IDE environment to allow users to build their topologies, insert easily the devices, and configure the required protocols and algorithms across the network. User can easily build his simulation network using three different models: Network Model, Node Model, and Process Model.
NS-3 [22] is a discrete event computer networks simulator used as real time network emulator. It is publicly available for research, and development. It is written completely in C++ and python. NS-3 is composed of several models: Mobility module, Event Scheduler Time Arithmetic, Node class, and Packets models.

QualNet is a network simulation software used to create virtual network that exhibits the behavior of real communication networks. It is a planning and testing tool to simulate complex and large scale communications networks [26]. Unlike other simulation software, QualNet is capable to incorporate the terrain in its simulation and it can be tested with the existence of different weather conditions. QualNet is composed of the following tools: QualNet andArchitect, QualNet Analyzer, QualNet Packet Tracer, QualNet File Editor, and QualNet Command Line Interface.

Omnet++ is a public-source, component-based, and modular simulation framework. It is mostly applied to the domain of network simulation and other distributed systems. The Omnet++ model is composed of hierarchically nested modules. The toplevel module is called the Network Module. This module contains one or more sub-modules each of which could contain other sub-modules.

Various Internet protocol model have been developed on the top of Omnet++ such as6LoWPAN Model, Network-of-Systems Simulator, NIST TESIMOMNeT++, and INET Framework [25]. The INET Framework is an open-source communication networks simulation package [25]. The INET Framework contains models for several wired and wireless networking protocols, including UDP, TCP, SCTP, IP, IPv6, Ethernet, PPP, 802.11, MPLS, OSPF, and many others [24]. It has number of modules which can be used by developers to build their simulation environment such as routers, switches, and hubs.

We have used in this paper INET simulator to conduct our experiments on Two-ray Multipath fading model. INET frameworks has provided the tools to simulate the fading between transmitting antenna and receiving antenna as well as the ability to change the data transmission rates using OFDM technique for wireless communication. INET framework has provided various path loss models:

a. Free space path loss.

b. Two-Ray Ground Reflection.

c. Rician Fading.

d. Log Normal Shading.

e. Two-Ray Interference. 


\section{SIMULATION RESULTS and DISCUSSIONS}

A simulation environment was implemented to study the effect of Two-ray Multipath fading model on OFDM. INET simulation framework and Omnet++ are capable to simulate transmission of data rates between devices up to $54 \mathrm{Mbps}$ at the $2.4 \mathrm{GHz}$ ISM band. We conducted our experiments on two wireless devices that communicate with each other whereas the distance between them was varying at each time the simulation is executed. The simulation parameter is shown in figure 1 which are stored in a file called omnetpp.ini.

A UDP packet was exchanged between the two devices and the size of the payload in the message was 2000 bytes. The simulation was repeated ten times to obtain the average values for our results. The distance between the transmitting and receiving antenna has changed at each run for a range from $5 \mathrm{~m}$ to $350 \mathrm{~m}$. The number of the received packets was measured at the receiver device at the end of the simulation period. Additionally, Signal to Noise ratio and Bit Error Rates were also measured at the receiving device. The experiment was conducted with IEEE 802.11g using OFDM technique for wireless communications with data rates: $54 \mathrm{Mbps}, 18 \mathrm{Mbps}$, and 6 Mbps.

Figure 2 shows the number of received packets at the destination device. It is obvious that there was not much difference with regard to the number received packets at the destination device whether the data rate was 54 or 18 Mbps. The increase in the Signal to Noise ratio caused the number of received packet to reach zero earlier with $54 \mathrm{Mbps}$ in comparison to other data rates.

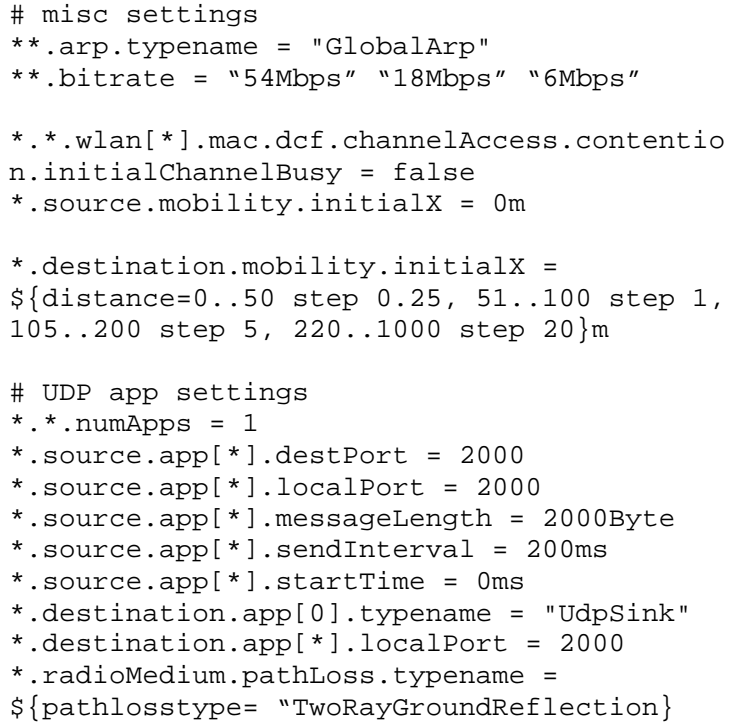

Figure 1: Simulation parameter in omnetpp.int used by INET framework.
For Signal to Nosie ratio, there was no change at the beginning as the distance between the devices started to increase as shown in figure 3 . We analyzed our results on Signal to Nosie ratio when the distance started to increase from 65 meter as shown in figure 4. For a distance of 100 meter and $54 \mathrm{Mbps}$, Signal to Nose ratio reached zero as well as the number of received packets reached zero. However, Signal to Nose ratio as well as the number of received packets reached zerofor the distance of 225 for 18 Mbps. Also, they reached zero when the distance between the two devices reached 350 meter for 6 Mbps.

Our observations on BER is shown in figure 5.For54 Mbps, the BER started to increase as the distance between the two devices reached 45 meter. For 18 Mbps, BER started to increase as the distance between the devices became 200 meter. For $6 \mathrm{Mbps}$, BER started to increase after the distance between the two devices reached 300 meters. Our results show that for OFDM with Two-ray Multipath fading, the highest data rate transmission is not always exhibiting better performance. Thus, it is better to move to the lower data from the beginning when the Multipath Fading Model exists at least to stay connected for longer distances as the Signal to Noise ratio drops when the distance between the two devices increases.

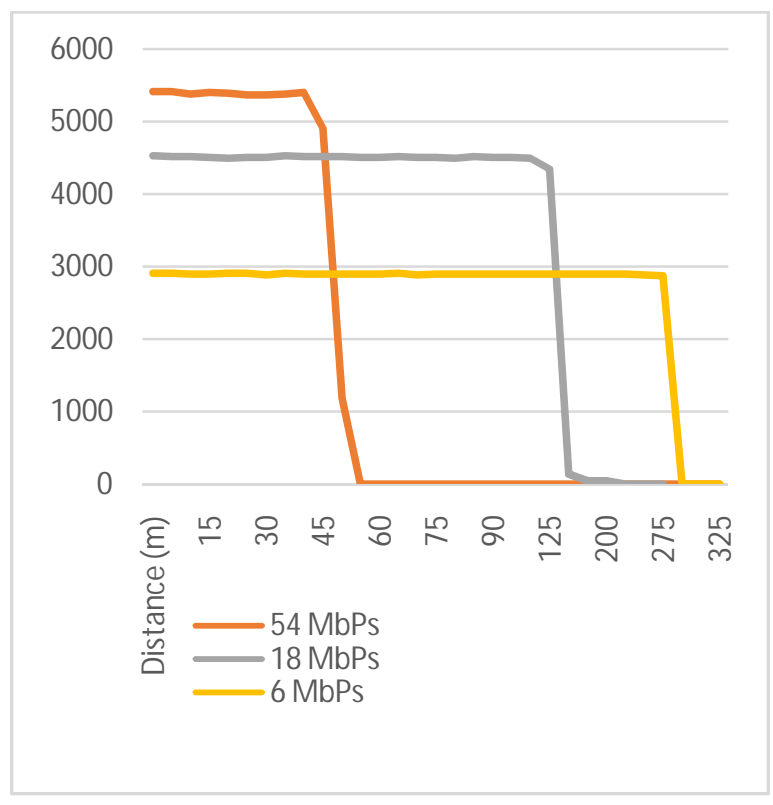

Figure 2: The number of received packets for OFDM with different data transmission rates. 


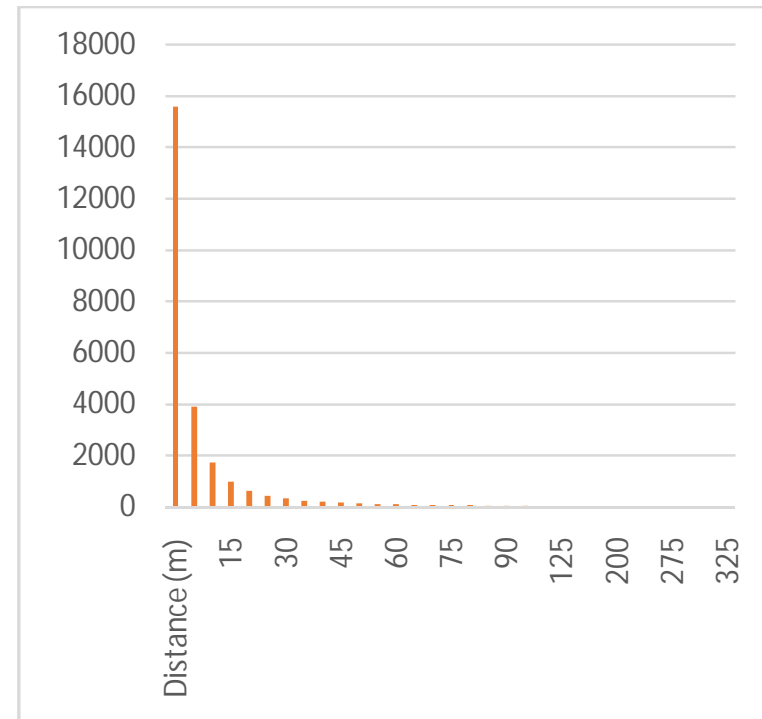

Figure 3: Signal to Noise ratio as the distance between varies for OFDM with different data transmission rates. At the beginning Signal to Noise ration was similar for OFDM with different transmission data rates.

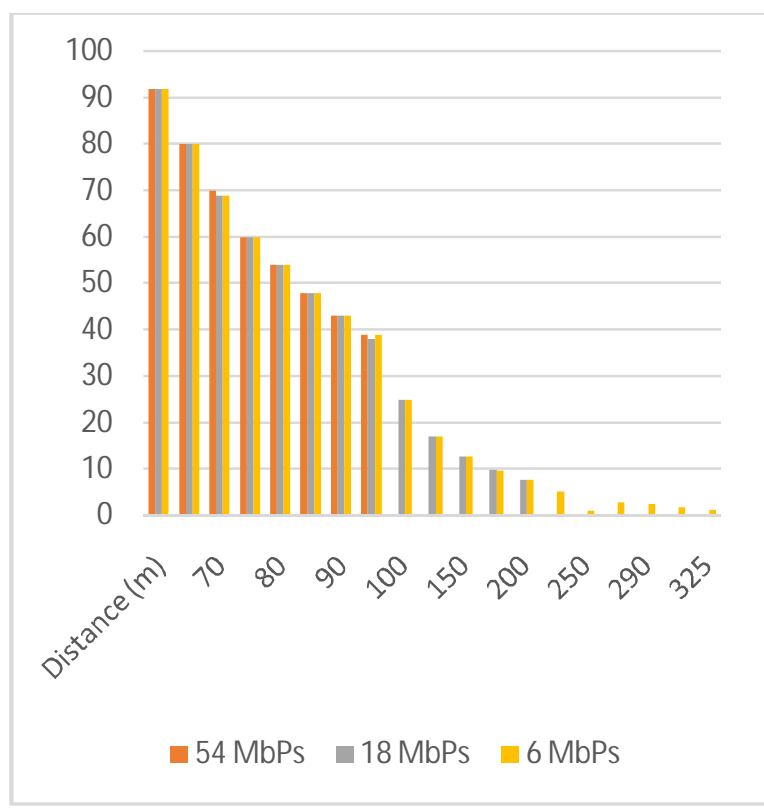

Figure 4: Signal to Noise ratio as the distance starts to increase form 65 meter.

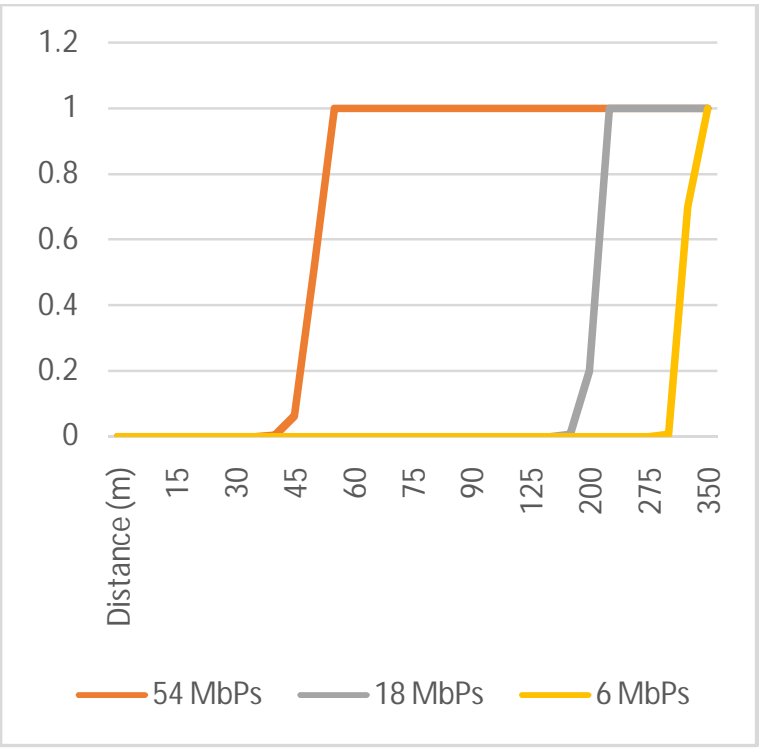

Figure 5:Bit Error Rates as the distance between the devices varies.

\section{CONCLUSIONS}

Fading is one of the major problems of digital communications, which results from different factors. This paper presented the effect Two-ray Multipath fading model on the IEEE 802.11g using OFDM with different transmission data rates. We found that high data rate transmission does not exhibit extremely high performance in an environment with Two-ray Multipath Model. It is possible for lower date rates to exhibit acceptable performance with regard to the number of the received packet at the destination host and much less BER. Thus, It is recommendable to move to the lower data from the beginning when experiencing Two-ray Multipath fading model with OFDM technique.

\section{REFERNCES}

1- Pei Zheng, Larry L. Peterson, Bruce S. Davie, and Adrian Farrel.Wireless Networking Complete,2nd Edition , Morgan Kaufmann, 2010.

2- L. Nathawad et al. An IEEE 802.11a/b/g SoC for Embedded WLAN Applications, 2006 IEEE International Solid State Circuits Conference - Digest of Technical Papers, San Francisco, CA, 2006, pp. 1430-1439.

3- Sang Lyul, Min Joong Soo, M Yujin Lim, and Jesung Kim. Performance evaluation of the Bluetooth-based public Internet access point, Information NetworkingProceedings, 15th International Conference on, 2001.

4- IEEE Standard for Information Technology Part 15.4: Wireless Medium Access Control (MAC) and Physical Layer (PHY) Specifications for Low-Rate Wireless Personal 
Area Networks (LR-WPANs), IEEE Standard 802.15.4 Working Group Std., 2003.

5- Lazarescu M.T. Wireless Sensor Networks for the Internet of Things: Barriers and Synergies, In: Keramidas G., Voros N., Hübner M. (eds) Components and Services for IoT Platforms. Springer, Cham. https://doi.org/10.1007/978-3-319-42304-3_9, 2017.

6- K. Romer and F. Mattern. The design space of wireless sensor networks,IEEE Wireles Comm., 11(6):54-61, December 2004.

7- Beena R. Ballal, Ankit Chadha, and Neha Satam. Orthogonal Frequency Division Multiplexing and its Applications, International Journal of Science and Research (IJSR), India Online ISSN: 2319-7064, 2013.

8- LQ. Lian, J., Gao, Y., Wu, P., Lian, D. Orthogonal Frequency Division Multiplexing Techniques Comparison for Underwater Optical Wireless Communication systems, Sensors 2019, 19, 160.

9- LQ.Khosla, D., Singh, S., Singh, R., and Goyal, S. (2010FDM Modulation Technique \& its Applications: A Review, International Conference on Innovations in Computing (ICIC 2017), pp:101-105.

10- Shuyu Zhang, Dobroslav Tsonev, Stefan Videv, Sanjay Ghosh, Graham A. Turnbull, Ifor D. W. Samuel, and Harald Haas. Organic solar cells as high-speed data detectors for visible light communication, Optica 2, 607-610 (2015).

11- F. Tosato, P. Bisaglia. Simplified Soft-Output Demapper for Binary Interleaved COFDM with Application to HIPERLAN/2,IEEE International Conference on Communications (ICC), vol. 2, 2002.

12- I. Gasulla, and J. Capmany. Transfer function of radio over fiber multimode fibre optic links considering third-order dispersion, Opt. Express, vol. 15, no. 17, pp. 10591-10596, 2007.

13- W. Y. Zou, and Y. Wu. COFDM: an overview, IEEE Trans. on Broadcasting, vol. 41, no. 1, pp. 1-8, 1995.

14- Smith, and David R.Digital Transmission Systems, Springer US, 2004.

15- Roger L. Freeman.Radio System Design for Telecommunications, 3rd Edition, Wiley-IEEE Press, 2007.

16- Bernard Sklar. Rayleigh fading channels in mobile digital communication systems Part I: Characterization, October 1997,IEEE Communications Magazine 35(9):136-146.

17- R. L. Olsen and B. Segal. New techniques for predicting the multipath fading distribution on VHF/UHF/SHF terrestrial line-of-sight links in Canada, Canadian Journal of Electrical and Computer Engineering, vol. 17, no. 1, pp. 11-23, Jan. 1992, doi: 10.1109/CJECE.1992.6593902.
18- Temidayo Omotosho.Impairment of radio wave signal by rainfall on fixed satellite service on Earth-space path at 37 stations in Nigeria, June 2009Journal of Atmospheric and SolarTerrestrial Physics 71(8-9):830-840.

19- B. H. Fleury and P. E. Leuthold. Radiowave propagation in mobile communications: an overview of European research, in IEEE Communications Magazine, vol. 34, no. 2, pp. 70-81, Feb. 1996, doi: 10.1109/35.481300.

20- Introduction to GloMoSim, LAP Lambert Academic Germany @2011 ISBN:3847323970 9783847323976.

21- Chen M., Miao Y., and Humar I. Introduction to OPNET Network Simulation,OPNET IoT Simulation. Springer, Singapore. https://doi.org/10.1007/978-981-32-9170-6_2.

22- iley G.F., Henderson T.R. The ns-3 Network Simulator. In: Wehrle K., Güneş M., Gross J. (eds) Modeling and Tools for Network Simulation, Springer, Berlin, Heidelberg. https://doi.org/10.1007/978-3-642-123313_2(2010).

23- OMNET++, https://omnetpp.org/, Last time visited $14^{\text {th }}$ of October 2020.

24- OPNET modeler, https://gonorthforge.com/opnet-modelercurrently-riverbed-modeler/, Last time visited $14^{\text {th }}$ of October 2020.

25- INET Framework, http://inet.omnetpp.org/, Last time visited $14^{\text {th }}$ of October 2020.

26- QualNet Simulator, https://www.scalablenetworks.com/products/qualnet-networksimulation-software-tool/, Last time visited $14^{\text {th }}$ of October 2020. 